\title{
Reducing cardiovascular mortality in chronic kidney disease: something borrowed, something new
}

\author{
L. Darryl Quarles, MD
}

Division of Nephrology, University of Tennessee Health Science Center, Memphis, Tennessee USA.

\begin{abstract}
Clinical vignette: A 48-year-old man with chronic kidney disease stage five due to type II diabetes mellitus and hypertension was referred for hemodialysis initiation. His physical exam showed a blood pressure of $150 / 80$, normal fundi, a positive fourth heart sound (S4), and trace pedal edema. Moderate aortic calcification was present on prior chest X-ray. The ECG showed left ventricle hypertrophy by voltage and slight prolongation of the QT interval. Medications included chlorthalidone, amlodipine, carvedilol, cholecalciferol, erythropoietin, and a phosphate binder. What additional therapy should be initiated to reduce vascular calcifications and cardiovascular mortality?
\end{abstract}

\section{Current therapy}

Cardiovascular disease (CV) is a major cause of morbidity and mortality in patients with chronic kidney disease (CKD), especially in those with end-stage renal disease (ESRD) undergoing thrice weekly hemodialysis (1). Indeed, the risk of CV-related mortality is 10 - to 20 -fold higher in hemodialysis patients. Interestingly, unlike the general population, in which coronary atherosclerotic disease is the principal cause of $\mathrm{CV}$ mortality, patients with CKD expire from chronic heart failure and sudden cardiac events. This is consistent with the fact that left ventricular hypertrophy (LVH) and vascular calcification are the most apparent cardiovascular abnormalities in patients with CKD (2). Many therapeutic strategies have failed to improve LVH, vascular calcifications, or survival in large-scale randomized controlled trials (RCTs) of patients with ESRD. Treatment with erythropoietin and statins and manipulation of hemodialysis prescription do not appear to lower mortality in ESRD $(3,4)$, nor do therapeutic strategies that address the mineral imbalances associated with CKD impact on $\mathrm{CV}$, including vitamin D therapy (PRIMO study), calcimimetics (EVOLVE study), phosphate binders, and calcium-free phosphate binders (4-6). Small RCTs evaluating

Conflict of interest: The author is a consultant for Amgen and Cytochroma and participates in the Amgensponsored DesignWrite speakers bureau.

Citation for this article: J Clin Invest. 2013; 123(2):542-543. doi:10.1172/JCI67203. angiotensin receptor blockers (7) and the nonselective $\beta / \alpha 1$-blocker carvedilol (8) suggest that these agents may reduce cardiovascular mortality in CKD, but these conclusions need to be substantiated in larger clinical trials. Nevertheless, activation of the renin-angiotensin-aldosterone system (RAAS) may contribute to LVH and myocardial fibrosis in CKD. It is unclear how RAAS is activated in CKD.

\section{Knowledge gap}

Simplistically, hypertension and increased arterial stiffness of calcified arteries should lead to LVH; however, the pathogenesis of LVH and vascular calcifications and their relationship to mineral metabolism perturbations are proving to be complex and refractory to current treatment strategies. A new idea is that chronic elevation of the bone-derived hormone FGF23 is associated with LVH and increased CV mortality in patients with CKD (9) and links abnormalities in mineral metabolism with adverse cardiovascular outcomes. The potential mechanisms whereby FGF23 is linked to CV mortality are uncertain. There is evidence that FGF23 activates FGF receptors (FGFRs) in the myocardium to stimulate LVH (10), but this direct mechanism is controversial because $\alpha$-Klotho, the FGF23 coreceptor required for FGFR activation, is not expressed in the myocardium. An equally likely possibility is that FGF23 activates FGFR/ $\alpha$-Klotho coreceptors in the kidney, which indirectly leads to LVH and cardiovascular mortality through acti- vation of the RAAS. In this regard, FGF23 appears to be a potent inhibitor of ACE2 expression in the kidney (11), which prevents degradation of Ang I and Ang II. This system is further impacted by treatment with $1,25(\mathrm{OH})_{2} \mathrm{D}$, which inhibits renin gene transcription and blocks RAAS but also stimulates FGF23 production in bone (12). The actions of $1,25(\mathrm{OH})_{2} \mathrm{D}$ to elevate circulating FGF23 levels may lead to conflicting effects on the myocardium, possibly explaining the inability of $1,25(\mathrm{OH})_{2} \mathrm{D}$ to improve LVH in the PRIMO study (5). Moreover, there is also evidence that Ang II suppresses renal $\alpha$-Klotho expression and that $\alpha$-Klotho overexpression can mitigate Ang II-induced proteinuria in experimental animal models (13).

\section{Research advances}

In this issue of the JCI, Voelkl and colleagues link elevated aldosterone levels with vascular calcification and provide a mechanism for RAAS activation and subsequent LVH in CKD (14). They report that, in klotho-hypomorphic $(k l / k l)$ mice, aldosterone directly stimulates vascular calcification by inducing PIT1-dependent phosphate transport in vascular smooth muscle cells via direct activation of the mineralocorticoid receptor. Importantly, the mineralocorticoid receptor antagonist spironolactone reduced vascular and soft tissue calcification and increased the life span in this model of $\alpha$-Klotho deficiency. These findings are consistent with several prior animal studies showing that aldosterone blockade affords vascular protection and reduces vascular calcifications through blood pressure-independent mechanisms (15-17).

Vascular calcifications are known to be associated with increased mortality, presumably through development of LVH in CKD; consequently, elevations of aldosterone in a $\alpha$-Klotho-deficient CKD model may also promote vascular calcification. These conclusions should be extrapolated to human CKD with some reservation. 
First, $\mathrm{kl} / \mathrm{kl}$ mice are a model of primary loss of $\alpha$-Klotho, and consequent elevations in FGF23 and $1,25(\mathrm{OH})_{2} \mathrm{D}$, which likely arise from end-organ resistance to FGF23, lead to severe hyperphosphatemia and hypercalcemia. In contrast, reductions in $\alpha$-Klotho in CKD are likely secondary to excess FGF23 and decreased $1,25(\mathrm{OH})_{2} \mathrm{D}$, both of which are known to suppress $\alpha$-Klotho expression in the kidney. Thus, the abnormalities of mineral metabolism and the mechanism of increased aldosterone may differ between $\mathrm{kl} / \mathrm{kl}$ mice and models of CKD. Nevertheless, these and other studies reporting that aldosterone targets vascular smooth muscle cells to stimulate vascular calcifications (15-17) suggest that aldosterone is the proximate mediator of cardiovascular and vascular toxicity in CKD.

\section{Recommendations}

Mineralocorticoid receptor antagonists are standard medical therapy for patients with moderate to severe heart failure symptoms and reduced left ventricular ejection fraction and for patients with heart failure after myocardial infarction in the general population. In addition, activation of the RAAS is profibrotic, whereas inhibition of this pathway is antifibrotic for the myocardium. Therefore, should we borrow this treatment for use in ESRD to treat LVH, hypertension, and vascular calcifications? Although hyperkalemia is a concerning complication of aldosterone antagonism in the setting of CKD, studies of chronic heart failure in patients with ESRD indicate that these agents may be used with close monitoring of serum potassium levels (18-21). The long-term outcomes of combined use of ACEI, angiotensin receptor blockers, and mineralocorticoid receptor antagonists in patients with ESRD have not been evaluated in rigorous, well-designed RCTs, thus the benefits and adverse consequences of single and combination therapies in this setting are unknown. The data supporting actions of both Ang II and aldosterone to stimulate cardiac and arterial blood vessel remodeling suggest that such trials may be warranted in ESRD (22).

Should we also consider new therapies to inhibit FGF23 to reduce LVH? Before recommending such therapy it will be necessary to confirm that FGF23 directly or indirectly stimulates myocyte growth leading to LVH in CKD. It will also be impor- tant to determine at what point during the course of progressive CKD that adaptive compensatory elevations of FGF23 become maladaptive. For example, treatment of animal models of CKD with neutralizing FGF23 antibodies increased rather than decreased mortality (23). Finally, the optimal range of serum FGF23 levels needs to be determined.

There are potential strategies to reduce FGF23 in CKD. In addition to blocking antibodies, FGFR inhibitors, which are being developed as cancer therapeutics, inhibit both the production (24) and end-organ effects of FGF23 (25). Serum FGF23 concentrations can also be reduced in ESRD by treatment with cinacalcet in combination with low-dose vitamin D analogues (26). Future trials are needed to explore multifaceted interventions geared toward improving survival by targeting the proximate pathophysiological factors responsible for increased cardiovascular mortality in CKD.

\section{Acknowledgments}

L.D. Quarles is supported by NIH grants DK095812, DK083303, and AR045955. The author would like to thank Csaba P. Kovesdy and Karl T. Weber for their helpful comments.

Address correspondence to: L. Darryl Quarles, University of Tennessee Health Science Center, 956 Court Ave., Suite B226, Memphis, Tennessee USA. Phone: 901.448.1759; Fax: 901.448.1175; E-mail: dquarles@uthsc.edu.

1. Foley RN, et al. Chronic kidney disease and the risk for cardiovascular disease, renal replacement, and death in the United States Medicare population, 1998 to 1999. J Am Soc Nephrol. 2005;16(2):489-495.

2. Foley RN, Parfrey PS, Harnett JD, Kent GM, Murray $\mathrm{DC}$, Barre PE. The prognostic importance of left ventricular geometry in uremic cardiomyopathy. J Am Soc Nephrol. 1995;5(12):2024-2031.

3. Besarab A, et al. The effects of normal as compared with low hematocrit values in patients with cardiac disease who are receiving hemodialysis and epoetin. N Engl J Med. 1998;339(9):584-590.

4. Moreno JA, et al. The inflammatory cytokines TWEAK and TNFalpha reduce renal klotho expression through NFkappaB. J Am Soc Nephrol. 2011;22(7):1315-1325.

5. Thadhani R, et al. Vitamin D therapy and cardiac structure and function in patients with chronic kidney disease: the PRIMO randomized controlled trial. JAMA. 2012;307(7):674-684.

6. Block GA, et al. Effects of phosphate binders in moderate CKD. J Am Soc Nephrol. 2012;23(8):1407-1415.

7. Cice G, et al. Effects of telmisartan added to Angi- otensin-converting enzyme inhibitors on mortality and morbidity in hemodialysis patients with chronic heart failure a double-blind, placebo-controlled trial. J Am Coll Cardiol. 2010;56(21):1701-1708.

8. Cice G, et al. Carvedilol increases two-year survivalin dialysis patients with dilated cardiomyopathy: a prospective, placebo-controlled trial. J Am Coll Cardiol. 2003;41(9):1438-1444

9. Gutierrez OM, et al. Fibroblast growth factor 23 and mortality among patients undergoing hemodialysis. NEngl J Med. 2008;359(6):584-592.

10. Faul C, et al. FGF23 induces left ventricular hypertrophy. J Clin Invest. 2011;121(11):4393-4408.

11. Dai B, et al. A Comparative Transcriptome analysis identifying FGF23 regulated genes in the kidney of a mouse CKD model. PLoS One. 2012;7(9):e44161.

12. Simpson RU, Hershey SH, Nibbelink KA. Characterization of heart size and blood pressure in the vitamin D receptor knockout mouse. J Steroid Biochem Mol Biol. 2007;103(3-5):521-524.

13. Mitani $\mathrm{H}$, et al. In vivo klotho gene transfer ameliorates angiotensin II-induced renal damage. Hypertension. 2002;39(4):838-843.

14. Voelkl J, et al. Spironolactone ameliorates PIT1-dependent vascular osteoinduction in klotho-hypomorphic mice. J Clin Invest. 2013;123(2):812-822.

15. Bellasi A, Ferramosca E, Ratti C, Block G, Raggi P. Cardiac valve calcification is a marker of vascular disease in prevalent hemodialysis patients. J Nephrol. 2012;25(2):211-218.

16. Wu SY, et al. Endogenous aldosterone is involved in vascular calcification in rat. Exp Biol Med (Maywood). 2012;237(1):31-37.

17. Nitta K, Akiba T, Nihei H. Aldosterone blockade and vascular calcification in hemodialysis patients. Am J Med. 2003;115(3):250.

18. Covic A, Gusbeth-Tatomir P, Goldsmith DJ. Is it time for spironolactone therapy in dialysis patients? Nephrol Dial Transplant. 2006;21(4):854-858.

19. Baker WL, White WB. Safety of mineralocorticoid receptor antagonists in patients receiving hemodialysis. Ann Pharmacother. 2012;46(6):889-894.

20. Taheri S, Mortazavi M, Pourmoghadas A, Seyrafian S, Alipour Z, Karimi S. A prospective double-blind randomized placebo-controlled clinical trial to evaluate the safety and efficacy of spironolactone in patients with advanced congestive heart failure on continuous ambulatory peritoneal dialysis. Saudi J Kidney Dis Transpl. 2012;23(3):507-512.

21. Bertocchio JP, Warnock DG, Jaisser F. Mineralocorticoid receptor activation and blockade: an emerging paradigm in chronic kidney disease. Kidney Int. 2011;79(10):1051-1060

22. Morishita Y, et al. Long-term effects of aliskiren on blood pressure and the renin-angiotensin-aldosterone system in hypertensive hemodialysis patients. Int J Nephrol Renovasc Dis. 2012;5:45-51.

23. Shalhoub V, et al. FGF23 neutralization improves chronic kidney disease-associated hyperparathyroidism yet increases mortality. J Clin Invest. 2012; 122(7):2543-2553.

24. Martin A, et al. Bone proteins PHEX and DMP1 regulate fibroblastic growth factor Fgf23 expression in osteocytes through a common pathway involving FGF receptor (FGFR) signaling. FASEB J. 2011;25(8):2551-2562.

25. Wohrle $\mathrm{S}$, et al. FGF receptors control vitamin D and phosphate homeostasis by mediating renal FGF-23 signaling and regulating FGF-23 expression in bone. J Bone Miner Res. 2011;26(10):2486-2497.

26. Wetmore JB, Liu S, Krebill R, Menard R, Quarles LD. Effects of cinacalcet and concurrent low-dose vitamin D on FGF23 levels in ESRD. Clin J Am Soc Nephrol. 2010;5(1):110-116. 